\title{
LASER AS A DEVICE FOR MEASUREMENT OF THIN THREADS - A SCHOOL LABORATORY EXPERIMENT IN PHYSICS
}

\author{
Hristina Deneva \\ Professional Technical High School "Doctor Nikola Vasiliady”, Bulgaria \\ Lyubomir Lazov \\ Erika Teirumnieka \\ Rezekne Academy of Technologies, Latvia
}

\begin{abstract}
The physics of the last century is included in all EU curricula and emphasis in education is shifted from content to the forms, methods and means of teaching and learning. Different kinds of lessons (including laboratory classes) and their didactic structure are subject to the understanding, adoption and creating conditions to build motivation to learn physics and astronomy, active utilization of physical knowledge and building cognitive and practical skills. Performance of demonstration and laboratory classes using a laser device is a type of teaching strategy to good education in physics. Properties of laser light as observation, classification, communication, drawing conclusions, planning, interpretation and forecasting, are particularly suitable when monitoring and studying the phenomena of interference and diffraction. Through both qualitative and quantitate ways, in this paper is presented a physical experiment for measurement of thin threads in a High school from students. Experimental skills will enhance interest in physics and especially to modern applications of laser devices, as well as career guidance of students.
\end{abstract}

Keywords: laser, laser beam, physical laboratory classes, diffraction, thin treads.

\section{Introduction}

Topicality for implementation on effective training of students in school physics and astronomy is not from today. On the one hand, it must meet the requirements of modern society which is mainly guaranteed by compulsory curriculum (Government Educational Requirements for Content, 2015). On the other hand are the requirements for permanent and thorough assimilation of physical knowledge and mastery of skills necessary in the implementation of educational and cognitive activity of students via continuously searching and evaluation of various forms of work organization, training methods and ways of active learning. 
Experiments have become an indispensable part of education. Indispensable part, because the combination of theoretical knowledge with experimental learning sessions has been ensured sustainable and successful learning.

According to curricula 10 class from Ministry of Education and Science (Government Educational Requirements for Content, 2015) in learning content VI. Observation, experiment, study and examination as expected results for students are included four Standards:

$\checkmark$ Standard VI.1. Perform observations and experiments as verified physical laws. A student has to perform independently simple physical experiments and explains the results.

$\checkmark \quad$ Standard VI.2. Perform laboratory experiment, process results and submit them tabular and graphical. Students have to demonstrate ability to work with laboratory experimental settings and appliances. As well as they have to use different methods for processing and presentation of experimental results.

$\checkmark$ Standard VI.3. Using simple physical and mathematical models, algorithms for solving problems and issues retrieve information from various sources, including by means of information technology. From the student is expected to solve quality problems and apply qualitative tasks and physical models in standard situations.

$\checkmark \quad$ Standard VI.4. Summarizes research results and draw conclusions about causality in physical phenomena. From the student is expected to demonstrate the ability to abstract thinking, the use of analogies and making generalizations.

Lasers such as widely used devices in technologies and the medicine are suitable to cover the above-mentioned expected results.

Of the 36 teaching hours in physics, two of them are studying lasers. The first one lesson is in topic "Sources of Light". In this topic with examples are illustrated important practical applications of thermal sources, of luminescence and the lasers. And in the second one, the theme "Nuclear transitions" is described the most important conditions for the generation of laser radiation.

Some authors (Black, 2005; Tippie \& Lee, 2016; Wojewoda, 2016) described that lasers are suitable to demonstrated phenomena as interference, reflection, refraction and diffraction. Mentioned phenomena are studied at the very beginning of the curricula in Physics and Astronomy 10. class (Maximov, 2001).

To explain interference and diffraction of light in the lesson is using Huygen's principle:

1. Each point on a wave front is the source of a spherical wavelet that spreads out at the wave speed. 
2. At a later time, the shape of the wave front is the line tangent to all the wavelets (Interference \& Diffraction, 2014).

Electromagnetic radiation propagates as a wave, and this is most strikingly seen with laser light, where light shining on a piece of paper looks speckled (with light and dark spots) rather than evenly illuminated, and where light shining through a small hole makes a pattern of bright and dark spots rather than the single spot we might expect from our everyday experiences with light.

This theoretical material from the curriculum without using a laboratory exercise is difficult interpreted and used by students. That is why in this paper we present a laboratory classes using laser light to investigate the phenomena of diffraction and will see how we can use these phenomena to make accurate measurements of very small objects like thin treads. From a methodological point of view the didactic structure of the laboratory exercise is represented by the scheme (figure 1):

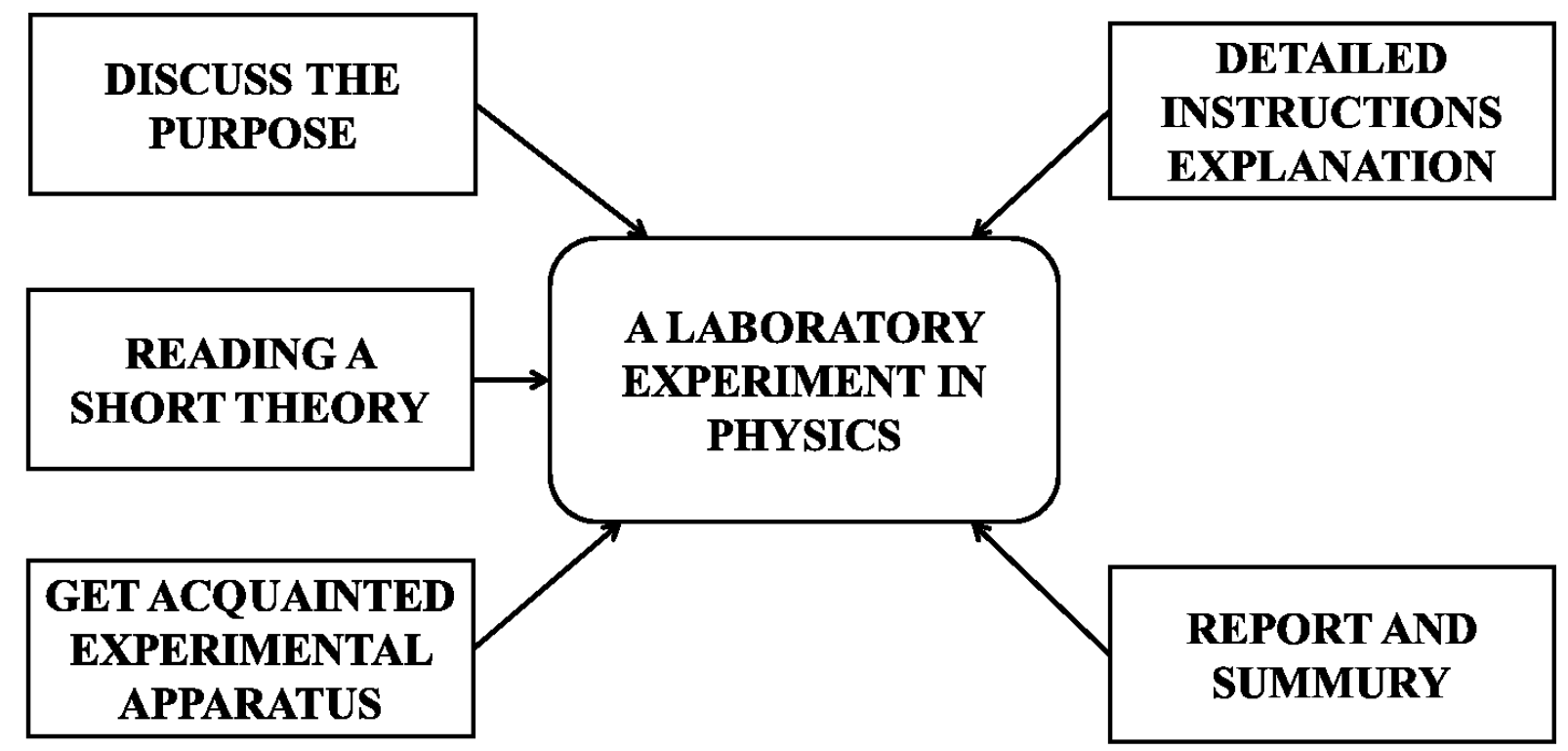

Figure 1 A didactic schema of the lab-experiment

\section{Pre-lab reading of short theory}

Diffraction is a phenomenon that occurs when electromagnetic wave meets on his way an obstacle. Depending on the coherence state of the light and according to the Huygens principle, "deviated" partial waves which interfere with each other and give rise to a diffraction pattern. A Babinet's Principle, which is not too difficult to derive, may be stated as follows:

"...the diffraction pattern from an opaque body is identical to that from a hole of the same size and shape except for the overall forward beam intensity." 
Thus, according to Babinet's Principle, cases A and B in Figure 2 will produce basically the same diffraction pattern. The only difference between them is that the beam passing around the obstruction will generally leave a bright extra spot at the centre of the screen (in case B, the central spot from the diffraction pattern may be too dim to see).

Except for the intensity of the central spot, the diffraction pattern produced by an opaque object in a wave train (or beam) is the same as that produced by an aperture of the same size and shape in an otherwise opaque screen.
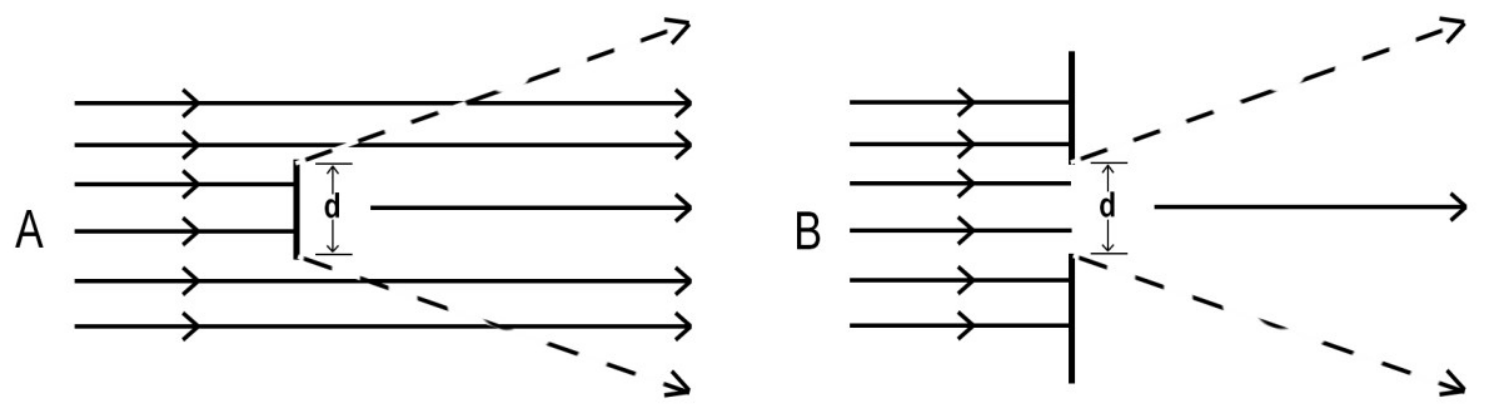

Figure 2 Two cases of beam passing around thin thread and a single slit

If the coherent, well-bundled light wave generated by a laser fall perpendicular to a thin wire the intensity distribution $I(\Theta)$ forming on the screen at a distance $L$ behind the thin thread (figure 3) will be determined by the function

$$
I(\Theta)=I(0) \cdot\left[\frac{\sin ^{2}\left[\frac{\pi d}{\lambda} \cdot \sin (\Theta)\right]}{\left[\frac{\pi d}{\lambda} \cdot \sin (\Theta)\right]^{2}}\right]
$$

and

$$
\sin \Theta=\frac{k \lambda}{d}
$$

where $\lambda$ is the laser wavelength; $k=0, \pm 1, \pm 2, \ldots$ is an integer called the order of the maximum.

$I$ (0) denotes the intensity maximum on the optical axis, and the angle between the normal to the end of the thread and the corresponding direction in which the diffractive waves are amplified i.e. direction of diffraction maximum of $k$-th order; See figure 4 of the experimental setup.

If the condition $I \gg>d^{2} / \lambda$ is satisfied it is called as Fraunhofer diffraction. 
Proceedings of the International Scientific Conference. Volume II, May $26^{\text {th }}-27^{\text {th }}$, 2017. 72-80

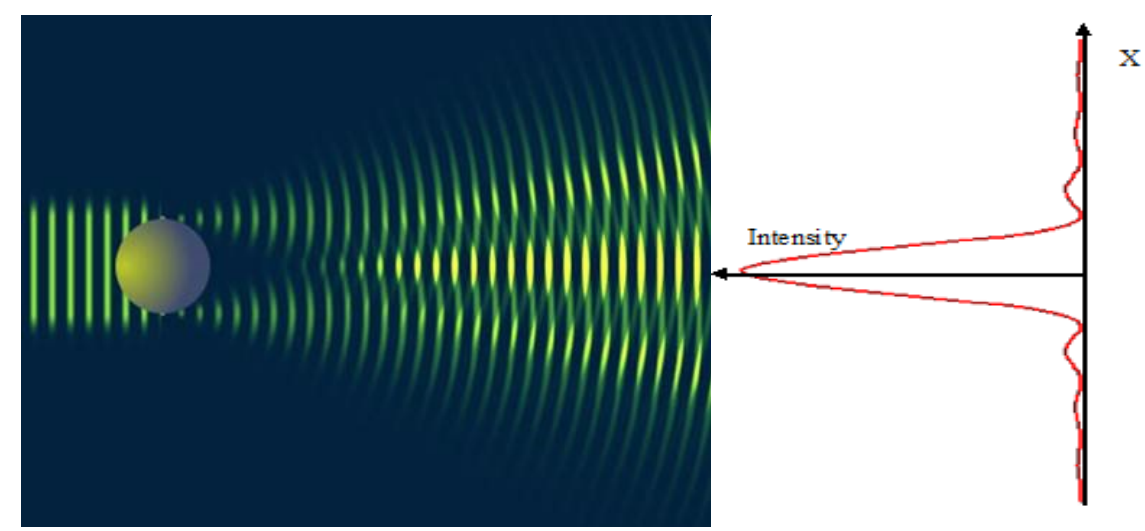

Figure $3 \mathrm{~A}$ view on the screen of intensity distribution

According to the Babinet principle, this formula (2) also applies to diffraction on a wire when the gap width $b$ is replaced by the wire thickness $d$.

If monochromatic light beam from a laser source falls perpendicularly on a thin thread thickness $d$ is obtained a diffraction pattern of alternating narrow light strips (diffraction maxima) - see figure 4. It is necessary to know that the condition for obtaining a diffraction maximum is expressed by the formula (2).

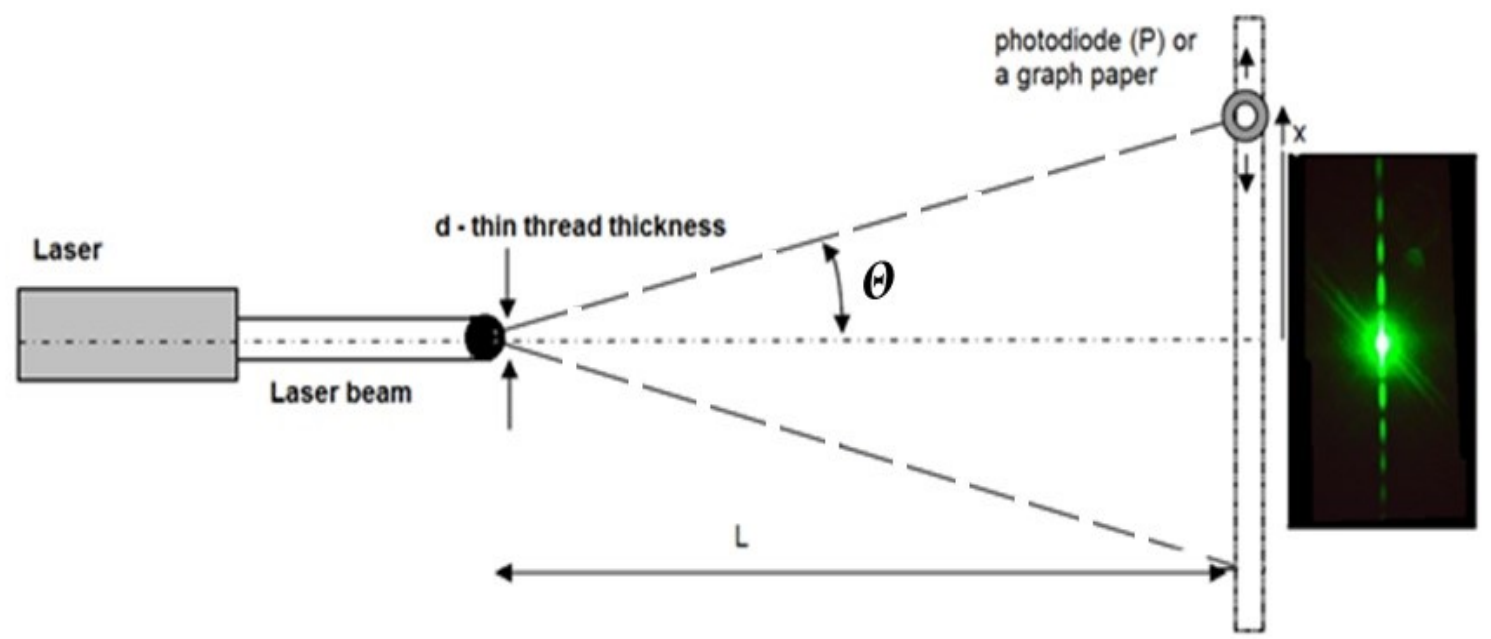

Figure 4 Diffraction maximums and minimums

If with an $\mathrm{L}$ indicate the distance from the thin thread to the central maximum, and with $2 x$ - the distance of the two maximums from the same $k$-th order, there are valid inequalities for enough large distances L:

$$
\operatorname{tg} \Theta \approx \sin \Theta \approx \frac{x}{L}
$$

from where

$$
\frac{x}{L}=\frac{k \lambda}{d}
$$


Finally, the formula which is used by students to calculate the thread's thickness is:

$$
d=\frac{2 \cdot k \cdot \lambda \cdot L}{2 x}
$$

\section{Purpose of the laboratory classes}

To determine the thickness of thin treads using a laser beam.

\section{Apparatus to be used}

Experimental set includes a laser source with a wavelength of $532 \mathrm{~nm}$, thin threadc, a screen E with a slot and an optical rail with holders. The screen is precoated with graph paper in order to more clearly measured the distances from the central bright spot to respective maximums. For the experiment were also used laser protective goggles and a clamping frame with threads of different diameters.

Measurement process instead of graph paper on screen can be automated sensor (photo detector) and a recorder or PC, which draws a graph on a computer screen (graph of the distribution of the intensity of $\mathrm{x}$ ). To scan the diffraction pattern can be used a photodiode (type BPW 34), in front of which has a diaphragm (width $0.3 \mathrm{~mm}$ ). Also it is necessary to have a microamperemeter with which to measure the photocurrent from the receiver (photodiodes), because of photocurrent is proportional to the intensity of the diffraction pattern in the given point.

\section{Explanation of instructions in the experiment}

1. Experimental data $(k, L, x, \lambda)$ are affixed in pre outlined a Table.

2. The separate component parts of the experimental set are fitted together - they are presented on Figure 5.

Instructions to Students for carrying out the experiment when using the laser source:

a) Be careful when handling the laser beam:

- Use the protective goggles.

- Never look into the direct laser beam, into a reflection or a diffracted beam.

- Do not remove the laser from the holder.

- When inserting, adjusting and removing optical components into/ from the optical rail switch off the laser beam.

- Fix the adjusted components to avoid unintentional changes in the position.

- Do not place any objects in the beam path that are not part of the test arrangement.

as adjustment required:

b) The laser beam should be on the wire right in the middle. 
3. Students observe a diffraction pattern using the laser beam with a wavelength $\lambda=532 \mathrm{~nm}$.

4. Described above measurements are carried out and their values are affixed in the Table. For high accuracy the measurements have been made in different maxima repeating them five times. Students take the arithmetic average for the given measured value.

5. From the data in Table is calculated the average value of the thread's thickness $d$ using formula (5).

6. At the end results are represented via a percentage error that has occurred in measurements.

7. Finally, students analyze data, report and state of conclusion.

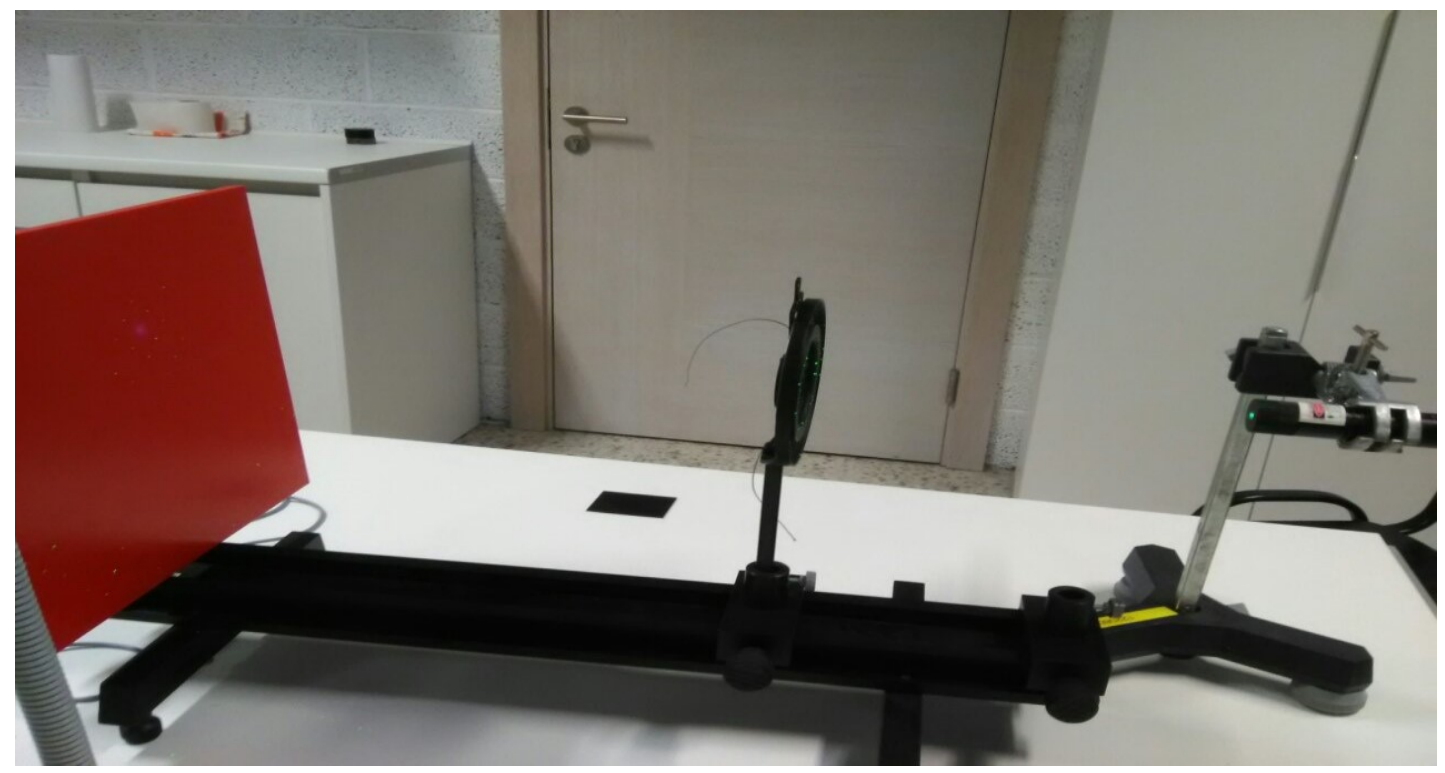

Figure 5 Basic parts of the experimental set: the laser source (right), thin treads (middle) and the screen (left)

With this laboratory exercise can be solved and inverse task: measuring the wavelength of the unknown laser light at a certain thickness of the thread or diffraction width of single slit.

This form of learning-cognitive activity is particularly enjoyable for students - they are most engaged. Although the teacher is a consultant, his task is much more difficult because:

- $\quad$ should oversee the organization and progress of the tasks;

- must guide the students and if necessary, supplement their emerging issues.

In conclusion of the lesson teacher can emphasize to students that diffraction-oriented methods are one of the common methods of quality 
assurance in the textile industry and in the metalworking industry. The on-line diameter control is regarded today as a necessity to get a satisfactory efficiency in the extrusion process. Likewise it is a must to guarantee the quality standard which is needed in an increasingly competitive market. The laser scanners, thanks to a perfect balance between ease of use and comprehensive functions, have proved to be very effective and suitable for extrusion automation. Just after the extruder head the product is still soft, can be easily deformed and in addition is moving at a speed that can attain remarkable values: in such conditions only instruments based on optical principles are able to check the dimensions without any risk of errors or damages due to the contact.

Using laser light, it has been possible to achieve reliable and accurate gauging, not affected by product movement and vibration.

The proposed laboratory experiment setup are simple and tasks are easy to realisation, the necessary equipment is relatively affordable (usually separate modules are already available) and easy to obtain. With featuring this equipment can be implemented more demonstrations and experiments than the ones mentioned in this article and the results are quite spectacular.

\section{Conclusion}

The laser incorporated in the laboratory can be used as a teaching and learning tool. In the context of optics, it can be used to measure incident, reflected and refraction angles, to build graphs and obtain relations between angles, and ultimately to determine the refractive index of optical media.

Therefore students can study the laws of optics and consolidate content taught in the classroom, such as lenses or diffraction and interference phenomena with an interactive methodology.

From everything said so far, we find it more than necessary to apply new approaches in education, because they help to build a student than proactive, creative, independent and self-monitoring personality. Even more now when we are from European family, the Bulgarian student must be can, knowledgeable, informed and confident in their abilities, because he will have to be competitive at some point with many professionals from other countries.

We would like to conclude with the words of Dr. Philippe Busquin European Commissioner for Research of the European Commission: "We must do more to raise public awareness of research in general and in particular to encourage young people to take an interest in science - not only as potential future scientists, technologists and technicians, but also as better-informed citizens." (Busquin, 2001). 


\section{References}

Black, T. (2005). Physics 102 Lab 8: Measuring wavelengths with a diffraction grating. Downloaded from http://web.physics.ucsb.edu/ phys128/experiments/interferometry/ measuring_wavelength.pdf

Busquin, P. (2001). European $S \& T$ Week 2000: Giving people a taste for science. Downloaded from https://cordis.europa.eu/pub/improving/docs/press_scienceweek_ 201000.pdf

Government Educational Requirements for Content, Ministry of Education and Science, Bulgaria, Downloaded (2016) from http://www.mon.bg/?go=page\&pageId=1\& subpageId=25, file://C:/Users/Deneva/Downloads/nrdb_2_00_uch_sadarjanie_pril5\% 20(1).pdf, http://www.mon.bg/?go=page\&pageId=1\&subpageId=28

Interference and Diffraction, Physics (2014). Downloaded from http://www.physics.umd.edu/ courses/Phys132/spring2014/LecturesA/A14WK14.pdf

Maximov, M. (2001). Physics and Astronomy, 10 class, Textbook, Sofia, publishing Bulvest (Bulgarian).

Tippie, Abb., \& Tammy, Lee (2008). Experiments with Diffraction. Downloaded from http://www.optics.rochester.edu/workgroups/berger/EDay/EDay2008_Diffraction.pdf

Wojewoda, G. (2016). Measuring index of refraction. Downloaded from http://www.pl.euhou.net/docupload/files/Excersises/WorldAroundUs/IndexOfRefractio $\mathrm{n} /$ IndexOfRefraction.pdf

Wojewoda, G. (2016). How to see that light is a wave - home laboratory of laser optics. Downloaded (2016) from http://www.pl.euhou.net/docupload/files/Excersises/World AroundUs/Diffraction/Diffraction_and_Interference_v2.pdf 